\title{
VISUAL FIELD INTERPRETATION WITH A PERSONAL COMPUTER BASED NEURAL NETWORK
}

\author{
ERKAN MUTLUKAN ${ }^{1}$ and DAVID KEATING ${ }^{2}$ \\ Glasgow'
}

\begin{abstract}
SUMMARY
The Computer Assisted Touch Screen (CATS) and Computer Assisted Moving Eye Campimeter (CAMEC) are personal computer (PC)-based video-campimeters which employ multiple and single static stimuli on a cathode ray tube respectively. Clinical studies show that CATS and CAMEC provide comparable results to more expensive conventional visual field test devices. A neural network has been designed to classify visual field data from PCbased video-campimeters to facilitate diagnostic interpretation of visual field test results by non-experts. A three-layer back propagation network was designed, with 110 units in the input layer (each unit corresponding to a test point on the visual field test grid), a hidden layer of 40 processing units, and an output layer of 27 units (each one corresponding to a particular type of visual field pattern). The network was trained by a training set of 540 simulated visual field test result patterns, including normal, glaucomatous and neuro-ophthalmic defects, for up to 20000 cycles. The classification accuracy of the network was initially measured with a previously unseen test set of $\mathbf{1 3 5}$ simulated fields and further tested with a genuine test result set of 100 neurological and 200 glaucomatous fields. A classification accuracy of 91-97\% with simulated field results and $65-100 \%$ with genuine field results were achieved. This suggests that neural networks incorporated into PC-based video-campimeters may enable correct interpretation of results in non-specialist clinics or in the community.
\end{abstract}

Perimetry is an indispensable part of complete examination of the visual system in ophthalmic or non-ophthalmic clinics such as neurology, neurosurgery, paediatrics and endocrine medicine. The use of perimetry is, however, severely restricted by its high cost, lack of widespread availability and the requirement of expert evaluation of the results. The Computer Assisted Touch Screen (CATS) ${ }^{1}$ and Computer Assisted Moving Eye Campimeter

From: 'Tennent Institute of Ophthalmology, University of Glasgow; ${ }^{2}$ Eye Department, Western Infirmary, Glasgow, UK.

Correspondence to: Erkan Mutlukan, MD, Henry Ford Hospital, Department of Ophthalmology, 2799 West Grand Boulevard, Detroit, MI 48202, USA.
$(\mathrm{CAMEC})^{2.3}$ are automated visual field analysers which operate on IBM-compatible standard personal computers (PC). These low-cost user-friendly video-campimeters have previously been shown to produce comparable results to more expensive conventional perimeters. In order to provide further assistance in diagnostic interpretation in CATS and CAMEC, a PC-based neural network for the pattern classification of the results was previously demonstrated using simulated visual field test results. $^{4.5}$

A neural network consists of a number of interconnected processing elements which are arranged in layers. Each processing element is analogous to a biological neuron. The input units correspond to the dendrites and the output units correspond to the axons. The network consists of three layers. The middle (= hidden) layer performs weighted summation of the input, applies a mathematical transfer function and allocates the input into one of the output categories. The network learns by example. The learning process involves presenting the network with an input pattern and a desired output pattern. Learning is accomplished by adjusting the input summation weights via repetitive cycling of the training patterns until the output accuracy approaches the desired level. ${ }^{6}$ The network can be trained for a pre-set number of cycles or until the output error falls below a threshold value. The ability of the neural network to classify the patterns is affected by the number of the processing elements in the hidden layer, the size of the training set of data and the number of recycles in the presentation of the training data.

This study describes the use of a neural network to perform pattern interpretation on genuine visual field data which can be generated by PC-based visual test devices.

\section{METHODS}

Neural-Works Professional II (Neural-Ware Inc., USA) software was used to design a neural network with 110 units in the input layer, each unit corresponding to a stimulus location on the central visual field test grid, ${ }^{7}$ a middle layer of 40 processing elements, and an output layer of 27 units that each corresponded to a particular type of sco- 


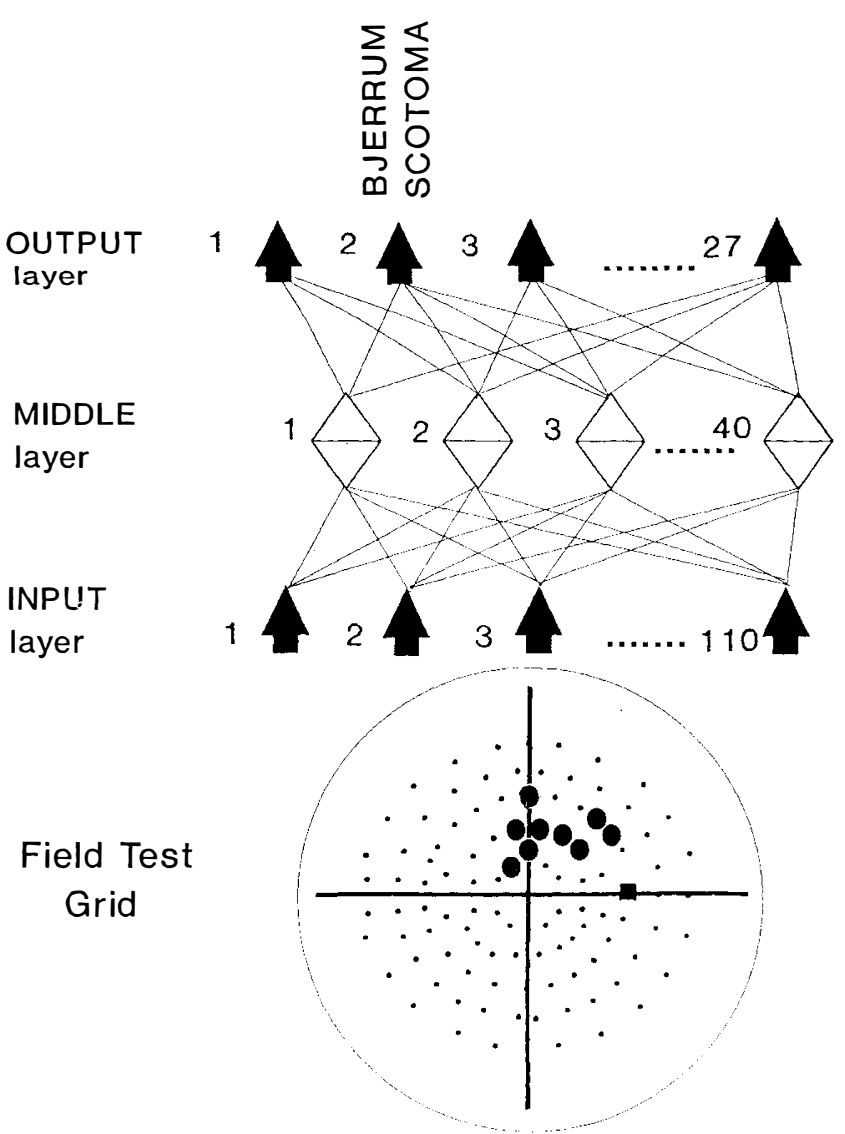

Fig. 1. A three-layer back propagation network. One hundred and ten input units constitute the test grid for central field. The large and small black dots represent 'missed' and 'seen' points in the central field test grid respectively. The black square represents the natural blind spot. The grid resolution of the input layer is 5-6 $6^{\circ}$ which is equal to that of comventional automated perimeters. ${ }^{7}$ Processing elements form the output by applying a mathematical transfer function to the summed input.

toma pattern, using an IBM-compatible 486 machine (Dell Inc.) (Fig. 1, Table I).

Six hundred and seventy-five visual fields ( 25 examples of each of the 27 visual field patterns) plotted on the grid constituting the input layer were used as the simulated data set to train and pilot test the network. The simulated field patterns satisfied the criteria for glaucomatous and neuro-ophthalmic visual field classification, that is, chiasmic defects show a correspondence to the vertical meridian, nerve fibre layer type defects to the horizontal meridian and isolated scotomas are contained within the central visual field (Fig. 2) ${ }^{8-11}$ The data were split into a training set of 540 plots and a test set of 135 randomly selected plots from the original data set.

The training session consisted of presenting the network with 20000 iterations of the training set. The distribution of the weight values and the output error were monitored throughout the learning process. Training was accomplished by successively adjusting the input weights on each presentation of the training pattern until the output error (= desired - actual accuracy) reached its lowest level.

Following the learning process, the network perform- ance was measured by presenting the test set and monitoring the output. The performance was further tested with 200 genuine visual field patterns from glaucomatous/glaucoma-suspect eyes and 100 visual field test results from eyes with neuro-ophthalmic problems. The actual visual field test results were obtained with conventional perimeters including the Humphrey visual field analyser, Friedmann analyser, Dicon autoperimeter, and Tübingen and Goldmann perimeters. Significant abnormalities on the field test plots within the central visual field $\left(30^{\circ}\right.$ from fixation) were converted into scotoma patterns and translated onto the input layer of the neural network using a mouse-driven digital drawing tablet and Harvard Graphics Draw-Partner software programs. At least two adjacent abnormal points in the input grid were regarded as a scotoma pattern.

\section{RESULTS}

When the performance of the neural network was examined using the simulated data test set $(n=135)$, a diagnostic accuracy of $91 \%$ and $97 \%$ was achieved according to the first and second choices made by the network.

When the genuine visual field data were presented to the network, $85 \%$ (254/300) of the perimetric results were recognised correctly as a result of the first output choice of the network. In the remaining 46 field results with inaccurate pattern recognition, the correct classification was the second choice of the network in 17 cases, improving the network accuracy to $90 \%(n=271 / 300)$. The full distribution of the genuine field patterns into different diagnostic categories and the accuracy of the network in each category are shown in Table II.

\section{DISCUSSION}

The use of neural networks in visual field interpretation has previously been experimented with in automated perimetry. ${ }^{+5.12 .13}$ The application of neural networks in personal computers and the systematic evaluation of their diagnostic accuracy according to different classes of genuine scotoma patterns, however, have not been reported before. The size of the training data set, the number of processing elements in the hidden layer and the number of recycles on network performance have been

Table I. Neural network output: 27 types

\begin{tabular}{|c|c|}
\hline $\begin{array}{l}\text { Bjerrum scotoma } \\
\text { Superior (SBS) } \\
\text { Inferior (IBS) }\end{array}$ & $\begin{array}{l}\text { Altitudinal } \\
\text { (complete or incomplete) } \\
\text { Superior (SA C/I) }\end{array}$ \\
\hline $\begin{array}{l}\text { Nasal step } \\
\text { Superior (SNS) } \\
\text { Inferior (INS) }\end{array}$ & $\begin{array}{l}\text { Inferior (IA C/I) } \\
\text { Paracentral scotoma } \\
\text { Generalised constriction }\end{array}$ \\
\hline $\begin{array}{l}\text { Quadrantanopia } \\
\text { (complete or incomplete) } \\
\text { Superior nasal (SNQ C/I) } \\
\text { Superior temporal (STQ C/I) } \\
\text { Inf erior nasal (INQ C/I) } \\
\text { Inf erior temporal }(\text { ITQ C/I ) } \\
\text { Central hemianopia }\left(<10^{\circ}\right) \\
\text { Nasal (NCH) } \\
\text { Temporal (TCH) }\end{array}$ & $\begin{array}{l}\text { Horizontal sectoranopia } \\
\text { Nasal (NHS) } \\
\text { Temporal (THS) } \\
\text { Hemianopia }\left(<30^{\circ}\right) \\
\text { (complete or incomplete) } \\
\text { Nasal (NH C/I) } \\
\text { Temporal (TH C/I) } \\
\text { Normal }\end{array}$ \\
\hline
\end{tabular}




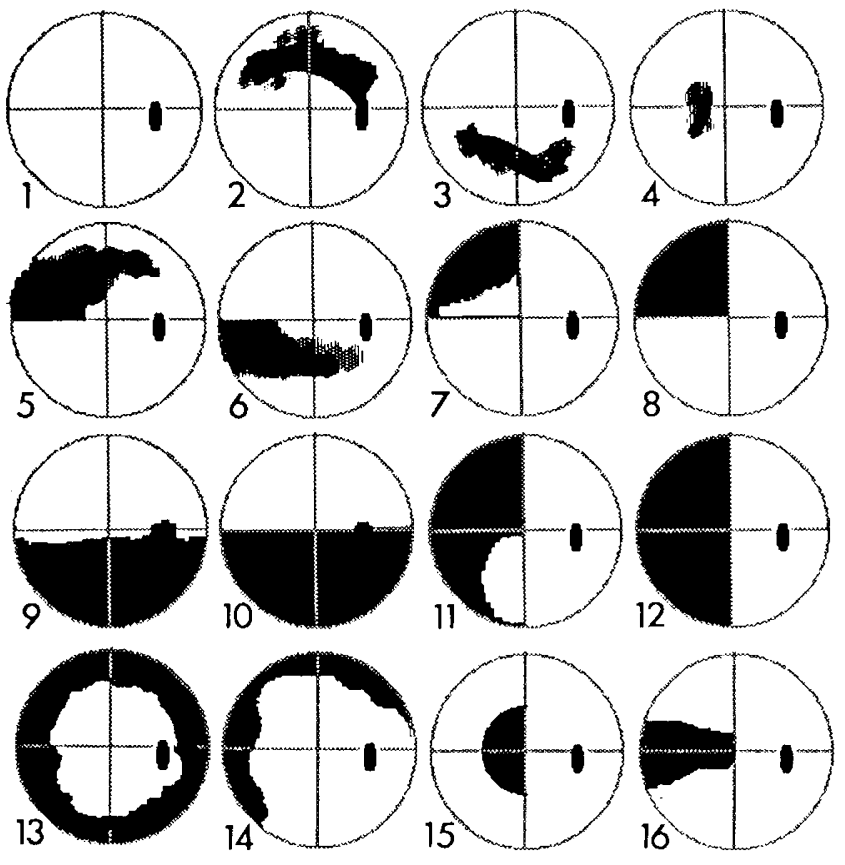

Fig. 2. Examples from simulated scotoma patterns which were used to train the network. 1 , normal; 2, superior bjerrum scotoma; 3 , inferior bjerrum scotoma; 4, paracentral scotoma; 5 , superior nasal step; 6, inferior nasal step; 7, quadrantanopia-incomplete; 8, quadrantanopia-complete; 9, inferior altitudinal - incomplete; 10, inferior altitudinal - complete; 11, hemianopia - incomplete; 12, hemianopia-complete; 13, constriction; 14, constriction; 15, central hemianopia; 16, hor izontal sectoranopia.

determined and adjusted as optimum in this study according to the results of our previous investigation..$^{5}$

The neural network successfully achieved a high accuracy in classification of previously unseen patterns. An average of 9 out of 10 genuine scotoma patterns were recognised correctly by the network. The field patterns that the network had most difficulty in classifying correctly, such as Bjerrum scotomas and nasal steps, indicate the need for further improvements in the network design. The subgroups of chiasmatic and nerve fibre layer type defects frequently misinterpreted by the network may, in future, be combined under a new and smaller number of output categories reflecting the general morpho-pathology of the visual damage. Output layer design with fewer and more comprehensive output types may improve the accuracy of the pattern classification. An alternative solution may be the initial quadrantic evaluation of the each field result fol-

Table II. Genuine visual field results: network classification accuracy $(n=300)$

\begin{tabular}{lrlrl}
\hline Diagnosis & \multicolumn{2}{c}{ First choice } & \multicolumn{3}{c}{ Second choice } \\
\hline Bjerrum scotoma & $65 \%$ & $(55 / 84)$ & $83 \%$ & $(70 / 84)$ \\
Constriction & $93 \%$ & $(43 / 46)$ & $98 \%$ & $(45 / 46)$ \\
Hemianopia & $89 \%$ & $(25 / 28)$ & $89 \%$ & $(25 / 28)$ \\
Nasal step & $79 \%$ & $(27 / 34)$ & $79 \%$ & $(27 / 34)$ \\
Quadrantanopia & $97 \%$ & $(31 / 32)$ & $97 \%$ & $(31 / 32)$ \\
Paracentral scotoma & $93 \%$ & $(13 / 14)$ & $93 \%$ & $(13 / 14)$ \\
Altitudinal def ect & $88 \%$ & $(15 / 17)$ & $88 \%$ & $(15 / 17)$ \\
Normal & $100 \%$ & $(45 / 45)$ & $100 \%$ & $(45 / 45)$ \\
Total & $85 \%$ & $(254 / 300)$ & $90 \%$ & $(271 / 300)$ \\
\hline
\end{tabular}

lowed by the sequential hemifield and total field assessments by the network as a step-by-step analysis approach.

The network training is performed in a few hours on a 486 personal computer. The software generates a run-time version of the trained network which can be transferred to less expensive personal computers and incorporated into PC-based video-campimetric test software for automatic reading of the individual results. The trained network can give instant classification of test data.

In conclusion, a three-layer propagation network can classify a wide range of visual field defects with an accuracy of $65-97 \%$. This addition to PC-based video-campimeters such as CATS and CAMEC will enable diagnostic interpretation of the visual field test results in non-ophthalmic clinics or in the community where perimetric expertise is not readily available.

This work has been supported by the International Glaucoma Association, the Royal National Institute for the Blind and the Royal Society of Medicine. The authors are grateful to Dr Gordon Dutton and Dr Bertil Damato for the purchase of the network software and the digital drawing tablet.

Key words: CATS, CAMEC, Neural network, Perimetry, Video-campimetry

\section{REFERENCES}

1. Mutlukan E, Keating D, Damato BE. A touch-screen multistimulus video-campimeter. In: Mills R (ed) Perimetry update 92/93. New York: Kugler-Ghedini, 1993:589-95.

2. Johnston SC, Damato BE, Evans AL, Allan D. Computerised visual field test for children using multiple moving fixation targets. Med Bio-Eng Comput 1989;27:612-6.

3. Mutlukan E. Computerised campimetry with static dark-onbright stimuli. Doc Ophthalmol 1993;84:335-50.

4. Keating D, Mutlukan E, Damato BE, Evans A. A back propagation neural network for the classification of visual field data. Invest Ophthalmol Vis Sci 1992;33:970.

5. Keating D, Mutlukan E, Evans A, McGarvie J, Damato BE. A back propagation neural network for the classification of visual field data. Phys Med Biol 1993;38:1263-70.

6. Rumelhart DE, McCleland JL. PDP Research Group: parallel distributed processing. In: Explorations in the microstructure of cognition. Vol. 1. Cambridge, Mass.: MIT Press, 1986.

7. Mutlukan E, Damato BE. Computerised perimetry with moving and steady fixation in children. Eye 1993;7:554-61.

8. Bajandas FJ, Lanning BJ. Neuro-ophthalmology review manual. Thorofare, New Jersey: Slack Inc., 1988.

9. Walsh TJ (ed). Visual fields: examination and interpretation. San Francisco: American Academy of Ophthalmology, 1990.

10. Harrington DO, Drake MV. The visual fields: text and atlas of clinical perimetry. St Louis, Mosby, 1990.

11. Beck RW, Smith CH. Neuro-ophthalmology: a problemoriented approach. Boston: Little, Brown, 1988.

12. Nagata S, Kani K, Sugiyama A. A computer assisted visual field diagnosis system using a neural network. In: Mills RP, Heijl A (eds) Perimetry update 91/92. New York, KuglerGhedini, 1991.

13. Keating D, Mutlukan E, Damato BE, Kirkness CM. Recognition of glaucomatous and neurological field loss with a neural network. Invest Ophthalmol Vis Sci 1993; (Suppl)34:1417. 